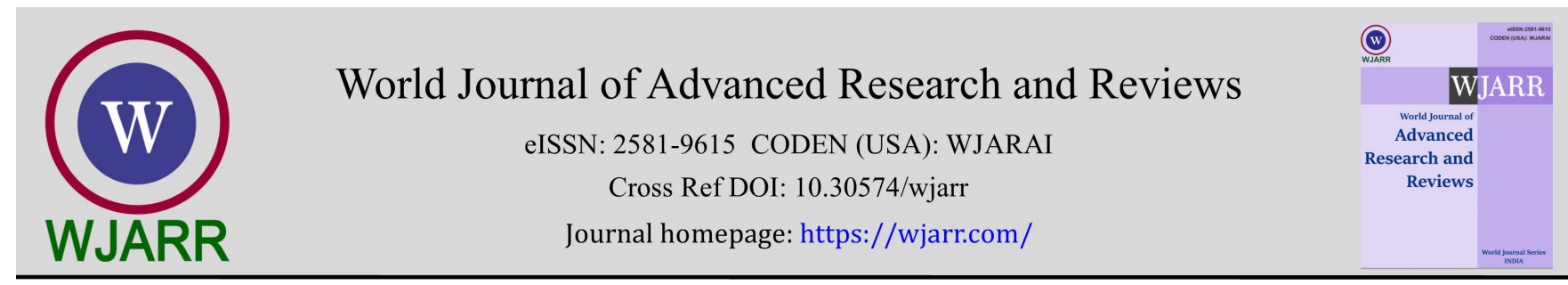

(RESEARCh ARTICLE)

Check for updates

\title{
Willingness to accept vaccination against SARS-cov-2: A survey of junior doctors
}

\author{
John Jude Kweku Annan 1, ${ }^{*}$, Betty Roberta Norman 2, Boniface Mensah ${ }^{3}$, Anthony Enimil ${ }^{4}$ and Collins \\ Kokuro ${ }^{5}$
}

${ }^{1}$ Lecturer \& Honorary Consultant, Department of Obstetrics and Gynaecology Komfo Anokye Teaching Hospital, School of Medicine and Dentistry Kwame Nkrumah University of Science and Technology, Kumasi, Ghana.

${ }^{2}$ Senior Lecturer Department of Internal Medicine Komfo Anokye Teaching Hospital, School of Medicine and Dentistry

Kwame Nkrumah University of Science and Technology, Kumasi, Ghana.

${ }^{3}$ Medical Officer Kumasi South Hospital P.O. Box 1908, Atonsu Agogo, Kumasi, Ghana.

${ }^{4}$ Senior Lecturer Department of Child Health Komfo Anokye Teaching Hospital, School of Medicine and Dentistry

Kwame Nkrumah University of Science and Technology, Kumasi, Ghana.

${ }^{5}$ Lecturer Department of Internal Medicine Komfo Anokye Teaching Hospital, School of Medicine and Dentistry Kwame

Nkrumah University of Science and Technology, Kumasi, Ghana.

World Journal of Advanced Research and Reviews, 2021, 09(03), 159-166

Publication history: Received on 06 February 2021; revised on 08 March 2021; accepted on 10 March 2021

Article DOI: https://doi.org/10.30574/wjarr.2021.9.3.0092

\begin{abstract}
Introduction: The Coronavirus disease 2019 (COVID-19) has become a cause of significant morbidity and mortality in the world. There are currently 4 COVID-19 vaccines have been developed for mass vaccination to combat the disease. Some people, however, have expressed safety and efficacy concerns with regards to the vaccines. In Ghana, junior doctors, being frontline doctors, are likely to first encounter patients presenting with COVID-19. They may also have the highest direct exposure to the virus even though their outcomes after infection may be good. The study sought to determine the potential acceptance rate of COVID-19 vaccine among junior doctors, the factors that will influence their acceptance and whether they will recommend the vaccine to their patients. Methods: The study was an ethically approved anonymous cross-sectional survey to determine the willingness to accept the SARS-CoV-2 vaccine among junior doctors in Ghana. The survey was designed using Google forms posted to the various junior doctor's social media group pages such as WhatsApp and Facebook with the study participants responding to 15 items. Results: Out of a total of 305 participants, females were 155 (50.8\%). Of the respondents, 171/305 (56.1\%) had cared for Covid-19 patients before, 51/305 (16.7\%) had had Covid-19 infection previously and 204/305 (66.9\%) will be willing to take the vaccine when available. Females were more likely to take the vaccine and those who were willing to accept the vaccine were more likely to recommend same to their family and others. Conclusion: This study has showed a more than average willingness to accept the COVID-19 vaccine. Female gender, living with the extended family and history of SARS-Cov-2 infection were positively associated with willingness to accept the COVID-19 vaccine. Doctors who were willing to accept the vaccine were also more likely to recommend their patients and family members to also accept the COVID-19 vaccine.
\end{abstract}

Keywords: COVID-19 vaccine; COVID-19; Coronavirus disease 2019; SARS-CoV-2; Severe Acute Respiratory Syndrome Coronavirus 2; Junior doctors.

\footnotetext{
${ }^{*}$ Corresponding author: John Jude Kweku Annan

Lecturer \& Honorary Consultant, Department of Obstetrics and Gynaecology Komfo Anokye Teaching Hospital, School of Medicine and Dentistry Kwame Nkrumah University of Science and Technology, Kumasi, Ghana.
}

Copyright (C) 2021 Author(s) retain the copyright of this article. This article is published under the terms of the Creative Commons Attribution Liscense 4.0. 


\section{Introduction}

The Coronavirus disease 2019 (COVID-19) outbreak was reported on December 302019 as a cluster of patients with pneumonia of an unknown aetiology in Wuhan City, Hubei Province, China [1]. The 2019 novel coronavirus was officially named Severe Acute Respiratory Syndrome Coronavirus 2 (SARS-CoV-2) by the International Committee on Taxonomy of Viruses [2]. The World Health Organization subsequently renamed the disease caused by SARS-CoV-2 from novel coronavirus to COVID-19 [3].

COVID-19 has spread rapidly across the globe causing devastating impact on public health services and loss of lives. The World health organization characterized the COVID-19 as a pandemic on the March 11, 2020 [4].

COVID-19 cases has continued to increase with daily mortalities and globally, as of 10:33am CET, 27 February 2021, there have been 112,902,746 confirmed cases of COVID-19, including 2,508,679 deaths, reported to WHO [5]. In Ghana there has been 82,586 confirmed cumulative cases with 594 cumulative deaths reported [6].

There are four categories of vaccines: Whole Virus (Sinopharm, Sinovac), Protein Subunit (Novavax) , Non-replicating Viral Vector (Oxford-AstraZeneca, Sputnik V) and RNA or mRNA (Pfizer-BioNTech, Moderna) [7, 8], all with the objective of producing immunity to the SARS-CoV-2 virus.

Globally there is now a mass vaccination campaign mainly in the developed world to combat the COVID-19 pandemic. Several countries including United kingdom, United States of America, Canada, Belgium, Canada, Chile, Argentina, Brazil, Chile, Germany, India, Russia, Belarus, Costa Rica, Croatia, Cyprus, China, The Czech Republic, Denmark, Finland, France, Greece, Hungary, Saudi Arabia, Israel, Italy, Kuwait, Malta, Mauritius, Mexico, Mauritius, Oman, Poland, Qatar, Romania, Serbia, Slovakia, Spain, Switzerland, Singapore, Turkey, United Arab Emirates among others have approved the administration of COVID-19 vaccinations for their populations [9]. There is very limited vaccine rollout in most African and other resource poor countries. Ghana is rolling out its COVID-19 vaccine campaign having received COVID-19 vaccine doses shipped via the COVAX Facility [10].

Even though the COVID-19 pandemic has caused significant mortalities and morbidities across the globe and overwhelmed global health care delivery, some people express concerns or fears with regards to the vaccines. These concerns involve the safety and efficacy of the vaccines. There has been various unfounded conspiracy theories and false information carried by the media especially social media about the SARS-CoV-2 vaccines and the vaccination process.

Studies on the willingness to accept the SARS-CoV-2 vaccine among the general public and various health worker groups has reported varying acceptance rates; 77.6\% in France [11], 65.7\% in Japan [12], 85•8\% in Australia [13], 54\% in Germany [14] and 69\% in the USA [15].

Willingness to accept vaccines is not static. People may change their positions on vaccines based on the information available to them. For example, as of January 2021, 54\% of Germans were definitely willing to get vaccinated against the coronavirus (COVID-19), however in November 2020, 37\% gave the same answer [14]. Germany began its coronavirus vaccination rollout shortly after Christmas 2020 with the BioNTech vaccine. A survey by the University of Zurich on vaccination attitudes, suggests the percentage likely or very likely to get vaccinated against the SARS-CoV-2 virus in Switzerland has risen from $41 \%$ to $50 \%$ [16].

A survey conducted by the Africa Centre for Disease Control and Prevention, in partnership with the London School of Hygiene \& Tropical Medicine across 15 African countries: Burkina Faso, Côte d'Ivoire, Democratic Republic of the Congo, Ethiopia, Gabon, Kenya, Malawi, Morocco, Niger, Nigeria, Senegal, South Africa, Sudan, Tunisia, and Uganda showed that a predominant majority (79\% average) of respondents in Africa would take a COVID-19 vaccine if it were deemed safe and effective(17). This survey showed significant variations in willingness across the African countries from $94 \%$ and 93\% in Ethiopia and Niger respectively to 65\% and 59\% in Senegal and the Democratic Republic of Congo respectively [17].

Another study from Congo however reported that only $27.7 \%$ of health care workers were willing to accept a COVID-19 vaccine if it was available [18]. There is paucity of information about the willingness of Ghanaians to accept the SARSCoV-2 vaccine.

Africans and people of African descent have a lot of mistrust towards vaccines and vaccinations due to historical experiences which were not so good. Rejection of vaccines has led to resurgence of some diseases for example Poliomyelitis in Nigeria $[19,20]$. 
Health workers are generally expected to be more knowledgeable with vaccines and having used various vaccines themselves and for their patients and seeing the positive results of disease prevention, their willingness and confidence in the vaccination to prevent COVID-19 will go a long way to improve the acceptance of the vaccine by the general public. If health workers have confidence in the vaccine, they are more likely to recommend it to the general public for good uptake in order to curb the menace of COVID-19.

In Ghana, junior doctors are the first front line doctors patients encounter at the outpatients and emergency rooms. They are therefore the most likely group of doctors to first see any patient coming in with COVID-19. They may also have the highest direct exposure to the virus even though their outcomes after infection may be good. The acceptability of these health workers of the COVID-19 vaccine may be an important indicator as to the young health work force and also determine whether they will recommend the vaccine to their patients.

The study seeks to determine the potential acceptance rate among these group of health care workers and the factors that will influence their acceptance of COVID-19 vaccine.

\section{Methods}

The study was an anonymous cross-sectional survey designed to look at the willingness to accept the SARS-CoV-2 vaccine among junior doctors in Ghana. The survey was designed using Google forms with the study participants responding to 15 items. The survey link was posted to the various junior doctor's social media group pages such as WhatsApp and Facebook. Informed consent was obtained individually by agreeing to be part of the study.

The study objectives were:

- $\quad$ Proportion of junior doctors in Ghana who will accept to be vaccinated against SARS-Cov-2

- Proportion of junior doctors in Ghana who will convince their patients to accept SARS-CoV-2 vaccine

- Various concerns/fears of SARS-CoV-2 among junior doctors in Ghana

- Factors affecting the willingness of a junior doctor to accept SARS-CoV-2 vaccine in Ghana

Participant's data regarding age, gender, religion, who they live with, number of people in their households, if they provide direct care to COVID-19 patients, history of COVID-19 infection, information regarding years of practice, and COVID vaccine acceptability questions such as "will you take the SARS-CoV-2 vaccine available to you?", "do you have concerns about the SARS-CoV-2 vaccine?" "What will motivate them to take the SARS-CoV-2 vaccine?" "will they encourage their family members or patients to take the vaccine", "do they have any comorbidities" was obtained.

The data obtained was exported to excel and analyzed using Rstudio software version 1.41103

We analyzed the distribution of the responses against the different questions using numbers and percentages. Associations between main outcome (will you take SARS-VoV-2 vaccine) and other independent variables were analyzed using generalized linear model and reported as Odds ratio with 95\% confidence intervals (95\% CI) and pvalues. Statistical significant was reported as p-value $<0.05$.

The study participants were junior doctors defined as doctors who have practiced for up to 5 years. Junior doctors in Ghana make up the majority of the number of medical doctors who serve as the first point of contact for a lot of patients. The perception of junior doctors on SARS-CoV-2 vaccine have repercussive effects on the fight against COVID-19 infection in Ghana. Participants answered the questions voluntarily and no personally identifiable information collected or stored.

Ethical approval was obtained from the Institutional Review Board for Research and Development (IRB/R\&D).

\section{Results}

A total of 305 participants had validated results available for analysis. Females were $155(50.8 \%)$. The majority were in the age range 25-30 years ,253 (83\%). Christians were 281 (92.1\%). Majority, 172 (56.4\%) had worked 1-3 years.

Participants who had cared for Covid-19 patients were 171 (56.1\%). Those who had had Covid-19 infection previously were $51(16.7 \%)$ and 204 (66.9\%) will be willing to take the vaccine when available. Those with co-morbid condition were $41(13.4 \%)$. 
Table 1 shows the association between those willing to take the vaccine against independent variables. Females were more likely to accept the vaccine. Those who were willing to accept the vaccine were more likely to recommend same to their family and others.

Reasons given for concerns raised about the vaccines were, it was developed too fast; vaccine contains microchip; I will have adverse reaction from vaccine.

The motivations for covid19 vaccine included: to protect myself, elderly parents, external family, and my patients. Others stated it will be needed for future travel.

Table 1 Comparing vaccine acceptability with independent variables among young Doctors

\begin{tabular}{|c|c|c|c|c|}
\hline & $\begin{array}{l}\text { Accept vaccine } \\
\mathrm{N}(\%)=204\end{array}$ & $\begin{array}{l}\text { Reject vaccine } \\
N(\%)=101\end{array}$ & OR $(95 \% \mathrm{CI})$ & p-value \\
\hline \multicolumn{5}{|l|}{ Gender } \\
\hline Female & $109(53.4)$ & $41(40.6)$ & \multirow{2}{*}{$1.68(1.04-2.72)$} & 0.036 \\
\hline Male & $95(46.6)$ & $60(59.4)$ & & \\
\hline \multicolumn{5}{|c|}{ Age range (years) } \\
\hline $18-24$ & $12(5.9)$ & $5(5.0)$ & ref & \\
\hline $25-30$ & $173(84.8)$ & $80(79.2)$ & $1.11(0.38-3.65)$ & 0.85 \\
\hline$>30$ & $19(9.3)$ & $16(15.8)$ & $2.02(0.59-6.96)$ & 0.26 \\
\hline \multicolumn{5}{|l|}{ Religion } \\
\hline Christians & $186(91.2)$ & $95(94.0)$ & $2.55(0.29-22.17)$ & 0.39 \\
\hline Muslims & $13(6.4)$ & $5(5.0)$ & $1.92(0.18-20.8)$ & 0.59 \\
\hline Others & $5(2.5)$ & $1(1.0)$ & ref & \\
\hline \multicolumn{5}{|c|}{ Household type } \\
\hline Nuclear & $110(53.9)$ & $65(64.4)$ & \multirow{2}{*}{$0.65(0.4-1.06)$} & 0.08 \\
\hline Extended & $94(46.1)$ & $36(35.6)$ & & \\
\hline \multicolumn{5}{|c|}{ Number per household } \\
\hline Up to 3 & $114(55.9)$ & $55(54.5)$ & \multirow{2}{*}{$1.06(0.66-1.71)$} & 0.81 \\
\hline More than 3 & $90(44.1)$ & $46(45.5)$ & & \\
\hline \multicolumn{5}{|c|}{ How many years have you being working (years) } \\
\hline$<1$ & $28(13.7)$ & $20(19.8)$ & ref & \\
\hline $1-3$ & $124(60.8)$ & $48(47.5)$ & $1.85(0.95-3.58)$ & 0.07 \\
\hline Above 3 & $52(25.5)$ & $33(32.7)$ & $1.13(0.55-2.31)$ & 0.74 \\
\hline \multicolumn{5}{|c|}{ Cared for covid19 patient } \\
\hline Yes & $110(53.9)$ & $61(60.4)$ & $1.23(0.66-2.29)$ & 0.52 \\
\hline No & $52(25.5)$ & $21(20.8)$ & $0.89(0.43-1.87)$ & 0.76 \\
\hline Unsure & $42(20.6)$ & $19(18.8)$ & ref & \\
\hline \multicolumn{5}{|c|}{ Have you tested positive } \\
\hline Yes & $36(17.6)$ & $15(14.9)$ & \multirow{2}{*}{$1.23(0.64-2.37)$} & 0.53 \\
\hline No & $168(82.4)$ & $86(85.1)$ & & \\
\hline
\end{tabular}




\begin{tabular}{|c|c|c|c|c|}
\hline \multicolumn{5}{|c|}{ Would you recommend vaccine for your family } \\
\hline Yes & $171(83.8)$ & $8(7.9)$ & $47.0(20.5-107.8)$ & 0.001 \\
\hline No & $3(1.5)$ & $27(26.7)$ & $0.24(0.07-0.87)$ & 0.029 \\
\hline Unsure & $30(14.7)$ & $66(65.4)$ & ref & \\
\hline \multicolumn{5}{|c|}{ Would you recommend vaccine for your patients } \\
\hline Yes & $176(86.3)$ & $17(16.8)$ & $25.8(13.3-50.2)$ & 0.001 \\
\hline No & $0(0)$ & $14(13.9)$ & - & 0.987 \\
\hline Unsure & $28(13.7)$ & $70(69.3)$ & ref & \\
\hline \multicolumn{5}{|c|}{ Any fears for the vaccine } \\
\hline Yes & $108(52.9)$ & $95(94.1)$ & \multirow{2}{*}{$0.07(0.03-0.17)$} & 0.001 \\
\hline No & $96(47.1)$ & $6(5.9)$ & & \\
\hline \multicolumn{5}{|c|}{ Co-morbidities } \\
\hline Yes & $30(14.7)$ & 11(10.9) & \multirow{2}{*}{$1.41(0.68-2.95)$} & 0.36 \\
\hline No & $174(85.3)$ & $90(89.1)$ & & \\
\hline
\end{tabular}

\section{Discussion}

With the COVD-19 pandemic claiming so many lives all over the world with over 2.5 million deaths [21], there is a need for a more effective solution to help reduce the incidence and mortality associated with the disease. Vaccination has shown over the years to be effective in reducing the incidence and even caused eradication of some diseases. The rate of acceptability of COVID 19 vaccine can massively influence the fight against this pandemic.

In our study, it showed $66.9 \%$ of junior doctors willing to accept the COVID 19 vaccine. This is lower compared to $78 \%$ acceptability rate among doctors in Israel [22] however, higher than $61.8 \%$ among family physicians in Malta [23]. This low level of acceptance may be attributed to various concerns raised about the vaccine. This includes fast rate of development of the vaccine, vaccine might contain a microchip and the fear of adverse reactions of the vaccines. Again, this observation may be explained by the perception of only getting a mild COVID-19 in younger population such as junior doctors compared to moderate and severe disease in older population. This observation has been noticed in several countries $[23,24]$. Junior doctors are also more likely to be influenced badly through misinformation about COVID-19 vaccines on various social media platforms [25] coupled with the high proportion (80\%) of patients with COVID-19 having a mild disease [26].

More female doctors (53.4\%) were willing to accept the vaccine compared to male counterparts (46.6\%). This finding was in contrast to that observed in other studies amongst healthcare workers in Israel, Kingdom of Saudi Arabia and United States of America [22, 27, 28]. These studies showed a positive correlation between males and the willingness to accept COVID-19 vaccine. They attributed this correlation to the increased severity and mortality of COVID 19 in male patients [29].

Junior doctors who lived with their nuclear families were less likely to accept COVID-19 vaccination (64.4\%) compared to the doctors who lived with their extended families (35.6\%). This result may be due to the fact that the young doctors who live with the aged grandparents and relatives would want to protect their relatives. This may be attributed to age being a risk factor for development of severe COVID-19 disease. Older patients are also at risk of co-morbidities including Diabetes mellitus, Hypertension and Chronic Kidney Disease. All these risk factors may increase the probability of a severe or even critical COVID-19.

Among junior doctors who had already had COVID-19, slightly higher percentage of them (17.6\%) were willing to accept a vaccine compared to their colleagues (14.9\%). This finding is consistent with the findings in a study conducted among health workers in Saudi Arabia [27]. The study also demonstrated a significant positive relationship between acceptance of COVID-19 vaccination and being previously infected with SARS-Cov-2. This observation may be due to the perceived risk of reinfection with SARS-Cov-2. Individuals who have been infected may want to prevent a reinfection and the perceived thinking that a second infection may be more severe. 
Another important observation made in this study was the high level of recommendation of the COVID-19 to patients $(86.3 \%)$ and family members $(83.3 \%)$ in doctors who are willing to take the vaccine. This same observation was seen in studies carried out among healthcare workers in France and United States $[11,28]$. Many studies have also proven that doctors who accept a vaccine are more likely to recommend their family members and patients to accept that vaccine $[30,31,32]$. With junior doctors and other healthcare workers willing to accept the COVID-19 vaccine, they are likely to advise their family members and patients to also accept the vaccine. Patients are more likely to take medical advice given to them by their doctors and this will help improve the acceptance of COVID-19 vaccine among the general population.

Fear of adverse effects of the vaccine contributed massively (94.1\%) to the rejection of the COVID-19 vaccine among junior doctors. This finding is consistent with findings from several studies as the fear of adverse effects of COVID-19 vaccines negatively influenced the acceptability of the vaccine $[13,22,27,33]$. The presence of co-morbidities also positively influenced the willingness to accept the COVID-19 vaccine as reported by other studies [28, 29]. With a strong link between co-morbidities and increased severity of COVID-19 and even mortality, it is not surprising that the presence of a co-morbidity will influence a person in accepting a COVID-19 vaccine [34, 35].

\section{Conclusion}

Junior doctors form an important group of healthcare workers in the fight against COVID-19 as they are at high risk of being infected with the SARS-Cov-2. Again, these doctors can play a major role in encouraging the general population to accept the COVID-19 vaccine.

This study has showed a more than average willingness to accept the COVID-19 vaccine. This can be massively improved with appropriate education among junior doctors and the entire population on the importance of the vaccine in the fight against the pandemic, its safety, benefits and the risks associated with the vaccine. This will go a long way to improve the acceptability of the vaccine in the general population as a majority of doctors who were unwilling to accept the vaccine had fear of adverse effects as their main reason.

Female gender, living with the extended family and history of SARS-Cov-2 infection were positively associated with willingness to accept the COVID-19 vaccine. Doctors who were willing to accept the vaccine were also more likely to recommend their patients and family members to also accept the COVID-19 vaccine.

\section{Compliance with ethical standards}

\section{Acknowledgments}

Our heartfelt appreciation and thanks goes to the junior doctors who participated in this study for their cooperation and willingness to be part of the research.

\section{Disclosure of conflict of interest}

All authors declare no competing interest.

\section{Statement of ethical approval}

Ethical approval was obtained from the Institutional Review Board for Research and Development (IRB/R\&D).

\section{Statement of informed consent}

Informed consent was obtained individually by agreeing to be part of the study.

\section{References}

[1] Bogoch, Isaac I, Alexander Watts, Andrea Thomas-Bachli, Carmen Huber, Moritz U G Kraemer, and Kamran Khan. "Pneumonia of Unknown Aetiology in Wuhan, China: Potential for International Spread via Commercial Air Travel." Journal of Travel Medicine. March 13, 2020; 27(2): taaa008. 
[2] Coronaviridae Study Group of the International Committee on Taxonomy of Viruses. The Species Severe Acute Respiratory Syndrome-Related Coronavirus: Classifying 2019-NCoV and Naming It SARS-CoV-2. Nature Microbiology. April 2020; 5(4): 536-44.

[3] Naming the coronavirus disease (COVID-19) and the virus that causes it [Internet]. [cited 2021 Feb 1].

[4] Cucinotta, Domenico, Maurizio Vanelli. WHO Declares COVID-19 a Pandemic." Acta Bio Medica Atenei Parmensis. March 19, 2020; 91(1): 157-60.

[5] WHO Coronavirus Disease (COVID-19) Dashboard [Internet]. [cited 2021 Feb 1].

[6] COVID-19 Updates | Ghana [Internet]. [cited 2021 Feb 27].

[7] The four types of COVID-19 vaccine - a snapshot [Internet]. Healthcare IT News. 2021 [cited 2021 Feb 27].

[8] There are four types of COVID-19 vaccines: here's how they work [Internet]. [cited 2021 Feb 27].

[9] Which countries have rolled out COVID vaccine? [Internet]. [cited 2021 Feb 1].

[10] COVID-19 vaccine doses shipped by the COVAX Facility head to Ghana, marking beginning of global rollout [Internet]. [cited 2021 Feb 27].

[11] Detoc, Maëlle, Sébastien Bruel, Paul Frappe, Bernard Tardy, Elisabeth Botelho-Nevers, and Amandine GagneuxBrunon. "Intention to Participate in a COVID-19 Vaccine Clinical Trial and to Get Vaccinated against COVID-19 in France during the Pandemic." Vaccine. October 2020; 38(45): 7002-6.

[12] Yoda, Takeshi, Hironobu Katsuyama. Willingness to Receive COVID-19 Vaccination in Japan. Vaccines. January 14,$2021 ; 9(1): 48$.

[13] Willingness to vaccinate against COVID-19 in Australia - The Lancet Infectious Diseases [Internet]. [cited 2021 Feb 1].

[14] COVID-19 vaccination willingness Germany 2021 [Internet]. Statista. [cited 2021 Feb 1].

[15] Reiter Paul L, Michael L. Pennell, Mira L Katz. Acceptability of a COVID-19 Vaccine among Adults in the United States: How Many People Would Get Vaccinated? Vaccine. September 2020; 38(42): 6500-6507.

[16] Covid: willingness to vaccinate rises to 50\% in Switzerland [Internet]. Le News. 2021 [cited 2021 Feb 1].

[17] Majority of Africans would take a safe and effective COVID-19 vaccine [Internet]. Africa CDC. [cited 2021 Feb 1].

[18] Nzaji MK, Ngombe LK, Mwamba GN, Ndala DBB, Miema JM, Lungoyo CL, et al. <p>Acceptability of Vaccination Against COVID-19 Among Healthcare Workers in the Democratic Republic of the Congo</p $>$ [Internet]. Vol. 11, Pragmatic and Observational Research. Dove Press. 2020 [cited 2021 Feb 1]; 103-9.

[19] Kapp Clare. Nigerian States Again Boycott Polio-Vaccination Drive. The Lancet. February 2004; $363,9410: 709$.

[20] Jegede, Ayodele Samuel. What Led to the Nigerian Boycott of the Polio Vaccination Campaign? PLoS Medicine. March 20, 2007; 4(3): e73.

[21] COVID 19 Map- Johns Hopkins Resource Center.

[22] Dror, Amiel A., Netanel Eisenbach, Shahar Taiber, Nicole G. Morozov, Matti Mizrachi, Asaf Zigron, Samer Srouji, and Eyal Sela. "Vaccine Hesitancy: The next Challenge in the Fight against COVID-19." European Journal of Epidemiology. August 2020; 35(8): 775-79.

[23] Grech, Victor, Jason Bonnici, Dorothy Zammit. Vaccine Hesitancy in Maltese Family Physicians and Their Trainees Vis-à-Vis Influenza and Novel COVID-19 Vaccination. Early Human Development. November 2020, 105259.

[24] Pedersen, Mogens Jin, Nathan Favero. Social Distancing during the COVID -19 Pandemic: Who Are the Present and Future Noncompliers? Public Administration Review. September 2020; 80(5): 805-14.

[25] McAteer, John, Inci Yildirim, Ann Chahroudi. The VACCINES Act: Deciphering Vaccine Hesitancy in the Time of COVID-19. Clinical Infectious Diseases. July 28, 2020; 71(15): 703-5.

[26] Coronavirus disease 2019 (COVID-19) Situation Report -46. World Health Organization.

[27] Qattan, Ameerah MN, Noor Alshareef, Omar Alsharqi, Naseem Al Rahahleh, Gowokani Chijere Chirwa, Mohammed Khaled Al-Hanawi. Acceptability of a COVID-19 Vaccine Among Healthcare Workers in the Kingdom of Saudi Arabia. Frontiers in Medicine. March 1, 2021; 8: 644300. 
[28] Shekhar Rahul, Abu Baker Sheikh, Shubhra Upadhyay, Mriganka Singh, Saket Kottewar, Hamza Mir, Eileen Barrett, Suman Pal. COVID-19 Vaccine Acceptance among Health Care Workers in the United States. Vaccines. February 2021; 9(2): 119.

[29] Galbadage, Thushara, Brent M. Peterson, Joseph Awada, Alison S. Buck, Danny A. Ramirez, Jason Wilson, and Richard S. Gunasekera. Systematic Review and Meta-Analysis of Sex-Specific COVID-19 Clinical Outcomes. Frontiers in Medicine. June 23, 2020; 7: 348.

[30] Paterson, Pauline, François Meurice, Lawrence R. Stanberry, Steffen Glismann, Susan L. Rosenthal, and Heidi J. Larson. Vaccine Hesitancy and Healthcare Providers. Vaccine. December 2016; 34(52): 6700-6706.

[31] Zhang Jing, Alison E. While, Ian J Norman. Nurses' Knowledge and Risk Perception towards Seasonal Influenza and Vaccination and Their Vaccination Behaviours: A Cross-Sectional Survey. International Journal of Nursing Studies. October 2011; 48(10): 1281-89.

[32] LaVela Sherri L, Bridget Smith, Frances M. Weaver, Marcia W. Legro, Barry Goldstein, and Kristin Nichol. Attitudes and Practices Regarding Influenza Vaccination Among Healthcare Workers Providing Services to Individuals With Spinal Cord Injuries and Disorders. Infection Control \& Hospital Epidemiology. November 2004; 25(11): 933-40.

[33] Daly M, Robinson E. Willingness to vaccinate against COVID-19 in the US: longitudinal evidence from a nationally representative sample of adults from April-October 2020. medRxiv. 2020.

[34] Wu, Zunyou, Jennifer M. McGoogan. Characteristics of and Important Lessons From the Coronavirus Disease 2019 (COVID-19) Outbreak in China: Summary of a Report of 72314 Cases From the Chinese Center for Disease Control and Prevention. JAMA. April 7, 2020; 323(13): 1239.

[35] Yang, Jing, Ya Zheng, Xi Gou, Ke Pu, Zhaofeng Chen, Qinghong Guo, Rui Ji, Haojia Wang, Yuping Wang, Yongning Zhou. Prevalence of Comorbidities and Its Effects in Patients Infected with SARS-CoV-2: A Systematic Review and Meta-Analysis. International Journal of Infectious Diseases. May 2020; 94: 91-95. 